\title{
Simplified Dual-Lumen Catheter-Facilitated Reverse Wire Technique for Markedly Angulated Collateral Channel Entry in Retrograde Chronic Total Occlusion Intervention
}

\author{
Kaize $\mathrm{Wu},{ }^{1} \mathrm{MD}$, Bingzheng Luo, ${ }^{2} \mathrm{MD}$, Zehan Huang, ${ }^{3} \mathrm{MD}$ and Bin Zhang, ${ }^{4} \mathrm{PhD}$
}

\begin{abstract}
Summary
The retrograde approach has significantly improved the success rates in complex coronary total occlusion (CTO) lesions. It has also become the predominant and important strategy in CTO recanalization. However, unsuccessful crossing of the collateral channels is the strongest predictor of retrograde failure, and adverse collateral channel morphology, including large channel entry angle, could reduce the success rate of collateral channel crossing. Reverse wire technique (RWT) was specifically developed for bifurcation lesions with an extremely angulated side branch, and nowadays, this can be achieved by the support of a dual-lumen catheter (DLC). We report a novel method named "simplified dual-lumen catheter-facilitated RWT" to facilitate markedly angulated collateral channel entry in retrograde CTO intervention. This new technique is simplified by making the reverse bend with the support of a DLC in the aorta instead of outside the guiding catheter, which is feasible, effective, and safe for markedly angulated collateral channel entry in retrograde CTO percutaneous coronary intervention $(\mathrm{PCI})$.
\end{abstract}

(Int Heart J 2021; 62: 416-421)

Key words: Crusade catheter, Coronary artery disease, Recanalization, Safety, Feasibility

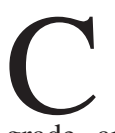

hronic coronary total occlusion (CTO) intervention remains one of the main challenges in percutaneous coronary intervention (PCI). The retrograde approach has significantly improved the success rates in complex CTO lesions and has also become the predominant and important strategy in CTO recanalization. ${ }^{1,2)}$ However, unsuccessful crossing of the collateral channels is the strongest predictor of retrograde failure. ${ }^{3)}$ Adverse collateral channel morphology, including large channel entry angle, could reduce the success rate of collateral channel crossing. Reverse wire technique (RWT) was specifically developed for bifurcation lesions with an extremely angulated side branch, ${ }^{4)}$ and nowadays, this can be achieved by the support of a dual-lumen catheter (DLC). ${ }^{5)}$ However, few data exist regarding RWT to support collateral channels crossing in retrograde CTO PCI.

Hence, in this study, we propose a novel method referred to as "simplified dual-lumen catheter-facilitated RWT" to facilitate markedly angulated collateral channel entry in retrograde CTO intervention. This new technique consists of the following three steps: (1) dragging the guiding catheter into the ascending aorta (AA) after inserting a guidewire (GW) into the main vessel (MV) (Fig- ure 1A); (2) advancing a DLC into the AA and inserting a guidewire into the AA by a length of $30 \mathrm{~mm}$ from the guidewire tip through the over-the-wire (OTW) port of the DLC (Figure 1B, C); (3) advancing the DLC into the MV to bend the guidewire in the AA with a reverse curve at a 30-mm point from the tip of the guidewire (Figure 1D); (4) simultaneously advancing the reverse wire (RW) and DLC into the distal MV (Figure 1E, F); and (5) pulling back the DLC and accurately inserting the RW into the target collateral channel (Figure 1G-J). Different from the conventional microcatheter-facilitated RWT, this new technique was simplified by achieving the reverse bend with the support of a DLC in the aorta instead of outside the guiding catheter. A representative case of this streamlined technique is reported as follows.

\section{Case Report}

A 54-year-old man who previously failed to recanalize RCA in-stent CTO was admitted to our hospital. The patient complained of severe angina, which seriously affected his quality of life. However, he refused to undergo coronary artery bypass grafting. As there is evidence that

From the ${ }^{1}$ Department of Cardiovascular Medicine, Affiliated Nanhai Hospital, Southern Medical University (People's Hospital of Nanhai District), Foshan, China, ${ }^{2}$ The Second School of Clinical Medicine, Southern Medical University, Guangdong Provincial People's Hospital, Guangdong Academy of Medical Sciences, Guangzhou, China, ${ }^{3}$ Department of Cardiovascular Medicine, The Second Affiliated Hospital of Guangzhou Medical University, Guangzhou, China and ${ }^{4}$ Department of Cardiology, Guangdong Cardiovascular Institute, Guangdong Provincial People's Hospital, Guangdong Academy of Medical Sciences, Guangzhou, China.

This study was supported by the Science and Technology Planning Project of Guangdong Province, Contract Grant Number: 2016 A020216022.

Address for correspondence: Bin Zhang, PhD, Department of Cardiology, Guangdong Cardiovascular Institute, Guangdong Provincial People's Hospital, Guangdong Academy of Medical Sciences, 96 Dongchuan Road, Guangzhou, 510100, China. E-mail: binzhang_cardio@163.com

Received for publication June 4, 2020. Revised and accepted October 7, 2020.

Released in advance online on J-STAGE March 6, 2021.

doi: 10.1536/ihj.20-391

All rights reserved by the International Heart Journal Association. 


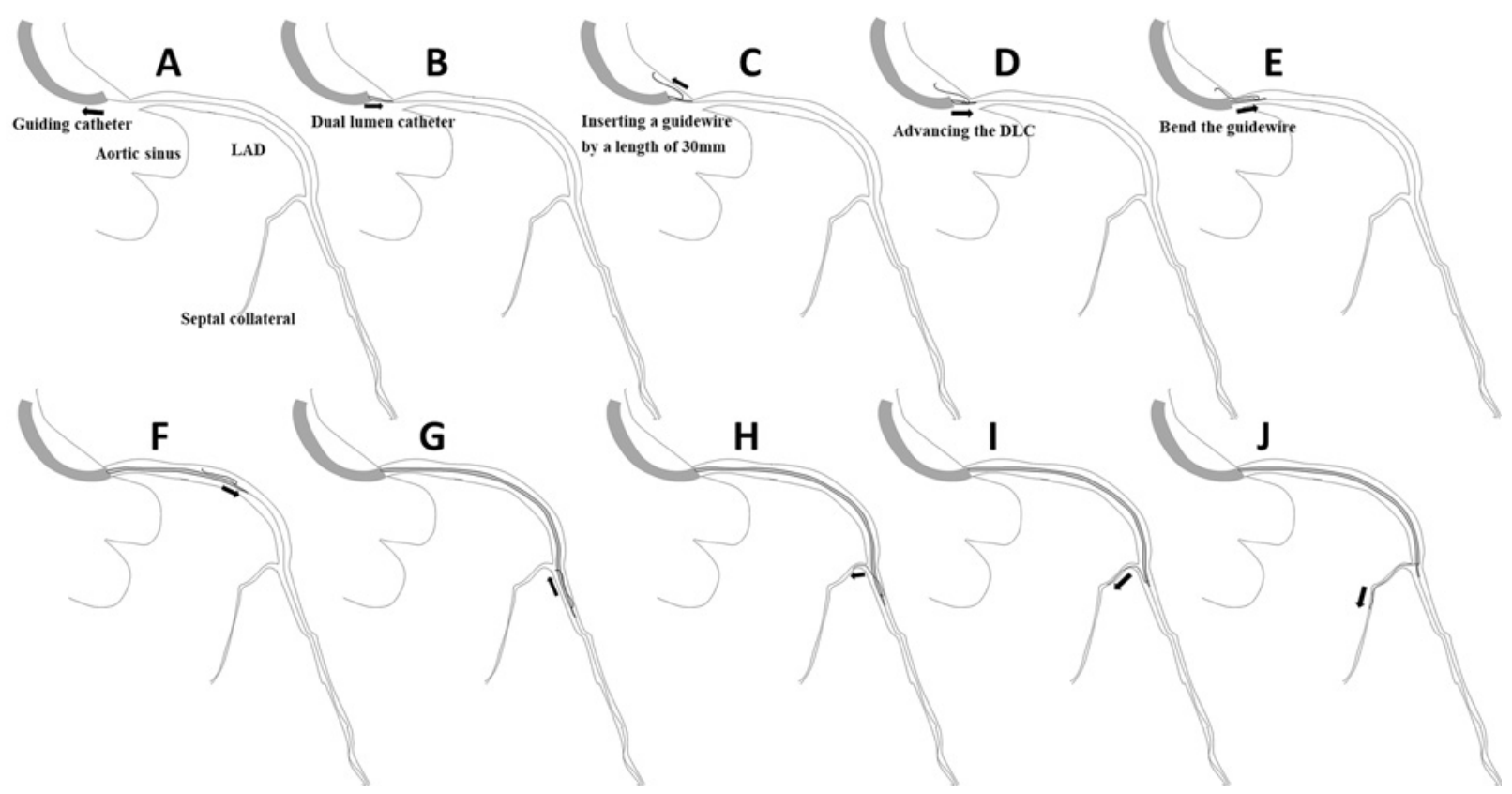

Figure 1. An illustration of the application of the simplified dual-lumen catheter-facilitated reverse wire technique. A: Inserting the guidewire into the main vessel (MV) and dragging the guiding catheter into the ascending aorta (AA). B: Advancing the dual-lumen catheter (DLC) into the AA. C: Inserting the guidewire into the AA by a length of $30 \mathrm{~mm}$ from the guidewire tip via the over-the-wire (OTW) port of the DLC. D-E: Advancing the DLC into the MV to bend the guidewire in the AA with a reverse curve at a 30-mm point from the tip of the guidewire. F: Simultaneously advancing the reverse wire (RW) and DLC into the distal MV. G-J: Pulling back the DLC and carefully inserting the RW into the target septal collateral channel.
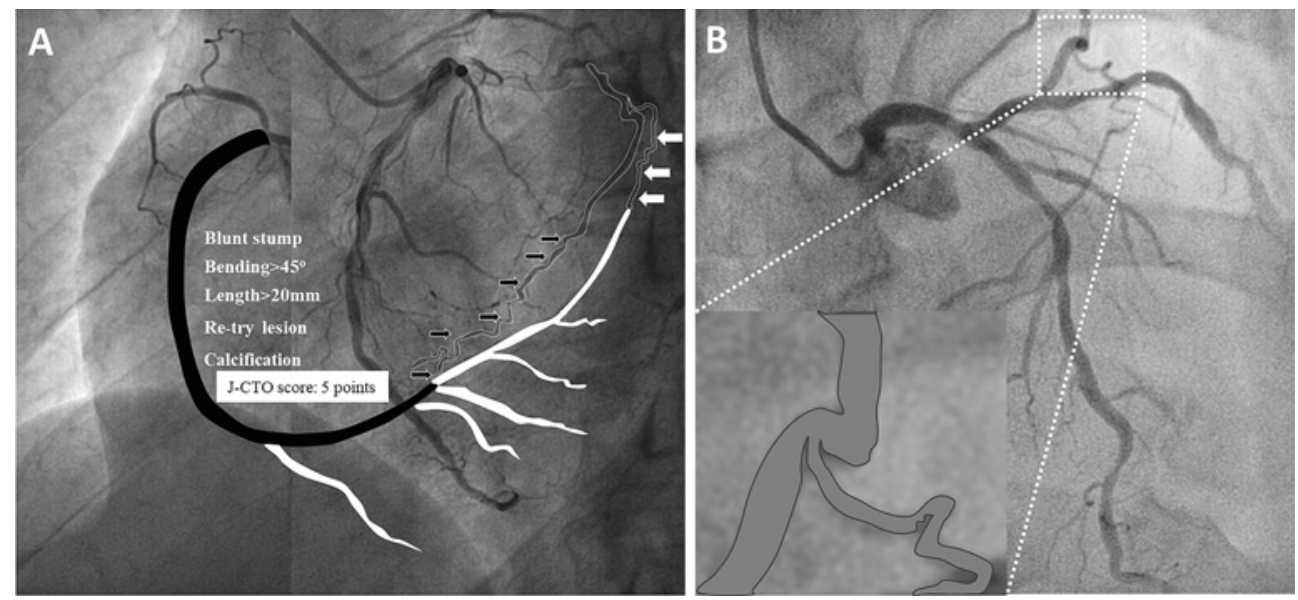

Figure 2. A: Coronary angiography demonstrated a proximal RCA in-stent CTO (J-CTO score of 5 points). An epicardial collateral from the atrioventricular branch of the circumflex artery (AVCx) to the posterolateral ventricular (PLV) branch of the RCA was favorable (indicated by a white arrow). B: The epicardial collateral branched from the AVCx with a markedly angulated entry is shown in the magnified image.

PCI for CTO revascularization can lead to a significant improvement in the health status of patients with stable angina, we performed PCI on the patient. ${ }^{6}$ His previous history included hypertension and diabetes mellitus. An electrocardiogram revealed ST-segment depression in the inferior leads, whereas an echocardiogram revealed satisfactory LV systolic function. Stress testing showed evidence of severe reversible myocardial ischemia in the inferior wall in the RCA territory. A coronary angiogram showed a proximal RCA in-stent CTO (J-CTO score of 5 points). An epicardial collateral from the atrioventricular branch of the circumflex artery (AVCx) to the posterolateral ventricular (PLV) branch of the RCA was favorable but with a markedly angulated entry (Figure 2). PCI via the retrograde approach was performed for the recanalization of the RCA in-stent CTO.

A 7-Fr AL1.0 guiding catheter was engaged to the RCA via the right radial artery and a 7-Fr XB3.5 guiding 

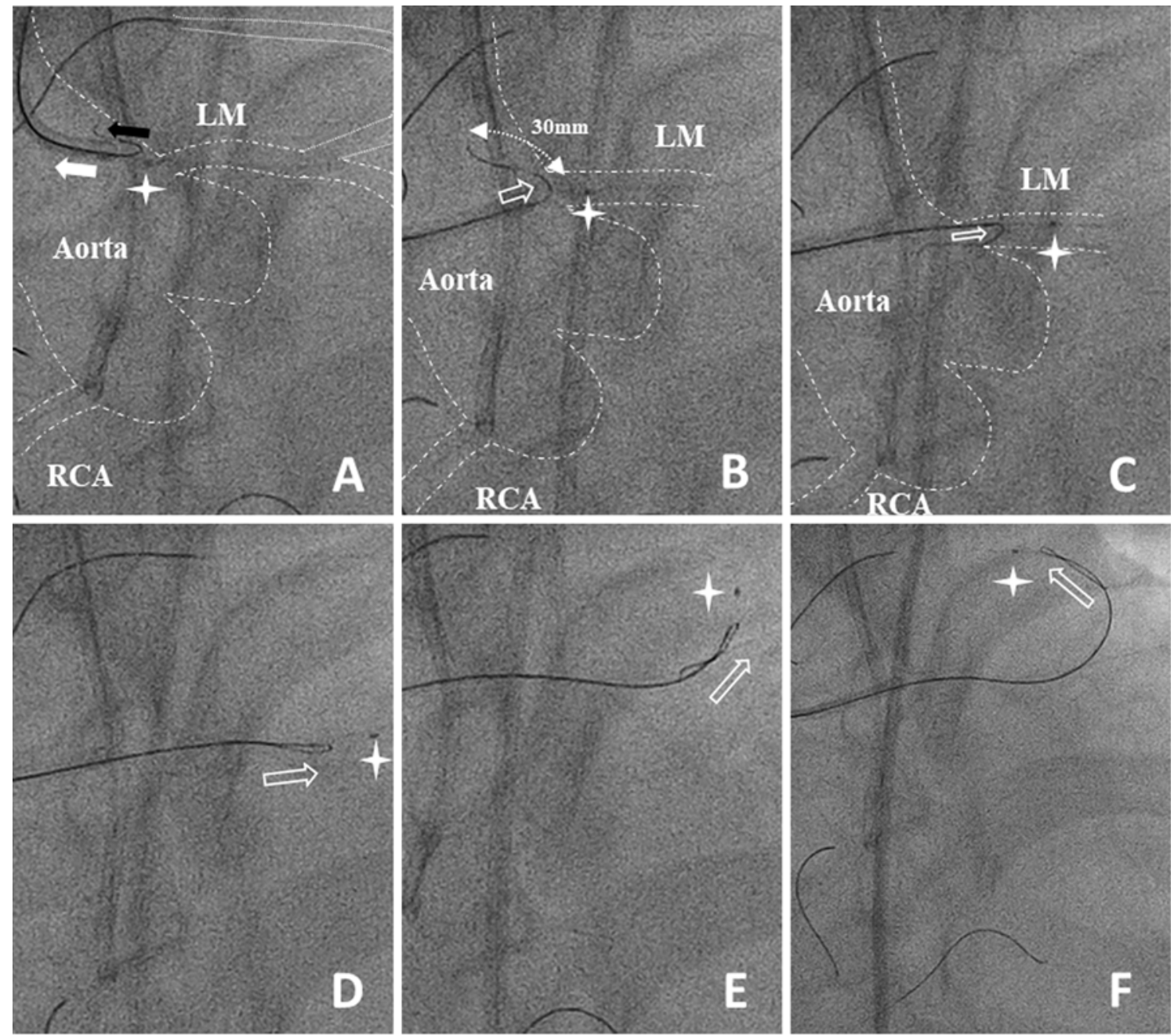

Figure 3. A: The Runthrough NS guidewire was inserted into the $\mathrm{AVCx}$, and the $\mathrm{XB} 3.5$ guiding catheter was dragged to the AA (indicated by a white arrow). B: The DLC (tip indicated by a white star) was advanced into the AA, and the Fielder XT-R (Asahi Intecc Co., Ltd., Aichi, Japan) was inserted into the AA by a length of $30 \mathrm{~mm}$ from the guidewire tip via the OTW port of the DLC (indicated by a white arrow). C: The DLC was advanced into the left main artery, and the Fielder XT-R was bent in the AA with a reverse curve at a 30-mm point from the tip of the guidewire (indicated by a white arrow). D-F: The Fielder XT-R and DLC were simultaneously advanced into the distal AVCx (indicated by a white arrow).

catheter to the left coronary artery via the right femoral artery. The antegrade wire was advanced into the false CTO segment. Subsequently, we initiated the retrograde approach via the selected epicardial collateral. Attempts to use a Sion black guidewire (Asahi Intecc Co., Aichi, Japan) to be advanced into the epicardial collateral were made but were unsuccessful. This is because of the challenging angulation between the AVCx and the epicardial collateral. Therefore, we attempted to perform "simplified dual-lumen catheter-facilitated RWT" to overcome this case of unusual anatomy.

First, a Runthrough NS guidewire was inserted into the AVCx, and the XB3.5 guiding catheter was dragged to the AA (Figure 3A). Next, a DLC (Crusade catheter; KANEKA Corp., Osaka, Japan) alone was advanced into the AA, and the Fielder XT-R (Asahi Intecc Co., Ltd., Aichi, Japan) was inserted into the AA by a length of 30 $\mathrm{mm}$ from the guidewire tip via the OTW port of the DLC
(Figure 3B). Then, the DLC was advanced into the left main artery, and the Fielder XT-R was bent in the AA with a reverse curve at a $30-\mathrm{mm}$ point from the tip of the guidewire (Figure 3C). Thereafter, the Fielder XT-R and DLC were simultaneously advanced into the distal AVCX (Figure 3D-F). The DLC was gently pulled back, and then the Fielder XT-R was also slowly withdrawn until the wire successfully entered the target epicardial collateral (Figure 4A-C). Subsequently, the DLC was changed to the microcatheter (FineCross, Terumo, Japan), and a guidewire (Sion black, Asahi Intecc Co., Ltd., Aichi, Japan) successfully tracked through the epicardial collateral (Figure 4D-F). The procedure was completed by the retrograde chronic total occlusion intervention (Figure 5). Finally, the RCA CTO was successfully recanalized without perforation of the epicardial collateral channel or other complications. 

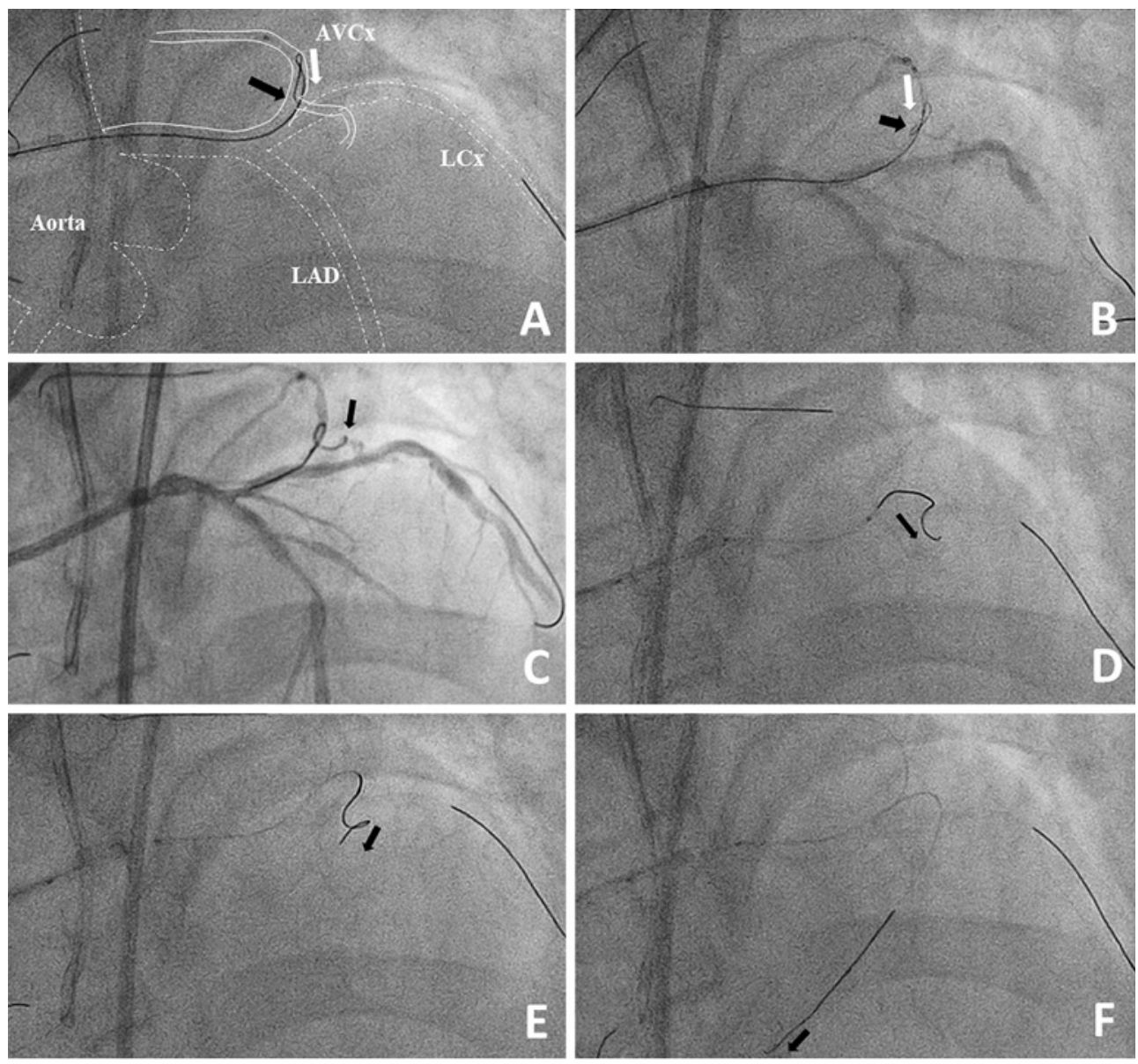

Figure 4. A-C: The DLC (indicated by a white arrow) was gently pulled back, and then the Fielder XT-R (indicated by a black arrow) was also slowly withdrawn until the wire entered the target epicardial collateral. D-F: The DLC was changed to the microcatheter (FineCross, Terumo, Japan), and a guidewire (Sion black, Asahi Intecc Co., Ltd., Aichi, Japan) (indicated by a black arrow) tracked successfully through the epicardial collateral.

\section{Discussion}

We present the use of a simplified dual-lumen microcatheter-facilitated RWT for facilitating markedly angulated collateral channel entry in retrograde CTO PCI. Nomura, et al. suggest the use of a polymer jacket hydrophilic-coated GW with a preshaped sharply curved tip for the dual-lumen microcatheter-facilitated RWT in PCI for markedly angulated bifurcated lesions.7) In addition, Canova, et al. described a similar case using a GW preshaped as a "swan neck" to engage the side-branch ostium. ${ }^{8)}$ The difference between the present case and the above cases is that, the polymer jacket hydrophilic-coated GW was directly bent in the aortic sinus by advancing the DLC to create a reverse curve shape at a $30-\mathrm{mm}$ point from the tip instead of preshaping the GW. Recently, Hasegawa, et al. have reported the "streamlined RWT" to overcome the difficulty in delivering RW beyond the severe stenosis of bifurcation, which is characterized by advancing a DLC alone beyond the stenosis and inserting a preshaped GW into a nontarget side branch distal to the bifurcation to make a reverse loop. ${ }^{9)}$ The differences between our method and this novel method are as follows:
(1) The "streamlined RWT" is mainly for complex bifurcation lesions, whereas the present method is for markedly angulated collateral channel entry in retrograde CTO intervention. (2) A preshaped RW outside the guiding catheter is not required. (3) After failed attempts with the polymer jacket hydrophilic-coated GW by the support of the DLC, the DLC and GW are withdrawn to the guiding catheter, and then the GW is directly bent in the aortic sinus by advancing the DLC to create a reverse loop at a 30-mm point from the tip. Consequently, the advantages of our method include convenience and efficiency. Therefore, this simplified RWT could help us save time and effort in accomplishing angulated collateral channel access in retrograde CTO PCI, even for the treatment of bifurcated lesions. Similar to the conventional microcatheterfacilitated RWT, ${ }^{5}$ the reverse bend is currently recommended to be made at a $3-\mathrm{cm}$ point from the guidewire tip. Moreover, a polymer jacket hydrophilic-coated guidewire is the optimal guidewire selection. In our case, the Fiedler XT-R wire (Asahi Intecc Co., Ltd., Aichi, Japan) was selected.

However, this technique has a disadvantage, i.e., making the reverse bend in the aorta and then advancing 

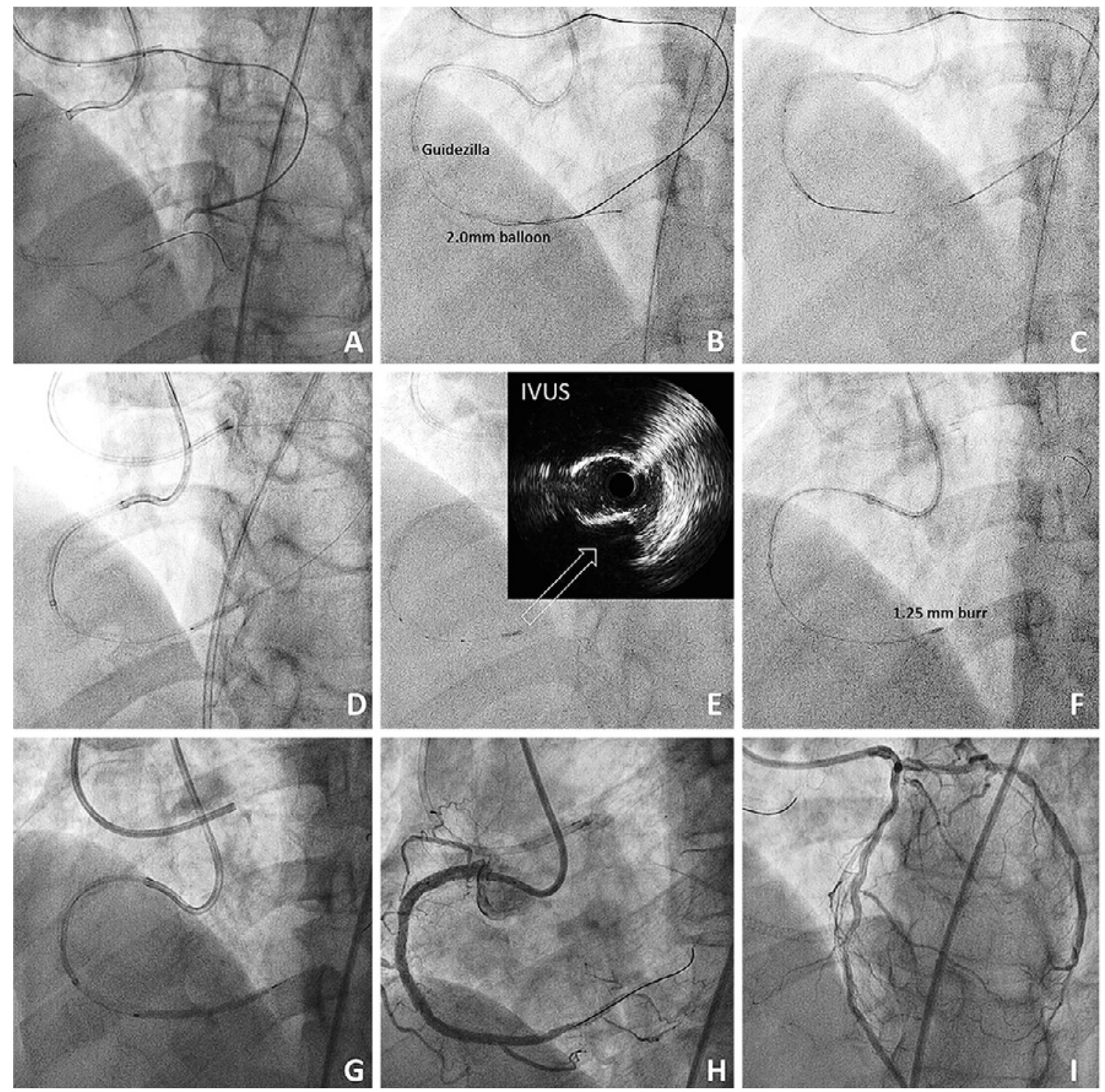

Figure 5. A: Tip injection from the retrograde microcatheter (Corsair, Asahi Intecc). B: The "Guidezilla reverse CART" technique was used for CTO crossing. C: The retrograde wire entered the Guidezilla followed by the retrograde microcatheter was advanced into the Guidezilla and externalization was finished by RG3 wire. D, E: A 2.0-mm balloon was inflated for intravascular ultrasound (IVUS) examination, which demonstrated a heavy calcification of the CTO lesion. F-I: After rotablation and implantation of four drug-eluting stents, the final angiography revealed a satisfactory result, and no perforation of the epicardial collateral channel was observed.

the DLC and the RW into the MV may result in the dissection of the donor vessel. Thus, awareness and vigilance of the potential dissection and skillful manipulation are required.

\section{Conclusion}

The simplified dual-lumen catheter-facilitated RWT is feasible, effective, and safe for markedly angulated collateral channel entry in retrograde CTO intervention.

\section{Acknowledgments}

We express our gratitude to all the coordinators who contributed to the recruitment in our center.

\section{Disclosure}

Conflicts of interest: Nothing to report.

\section{References}

1. Karmpaliotis D, Michael TT, Brilakis ES, et al. Retrograde coronary chronic total occlusion revascularization: procedural and in-hospital outcomes from a multicenter registry in the United States. JACC Cardiovasc Interv 2012; 5: 1273-9.

2. Tsuchikane E, Yamane M, Mutoh M, et al. Japanese multicenter registry evaluating the retrograde approach for chronic coronary total occlusion. Catheter Cardiovasc Interv 2013; 82: E654-61.

3. Rathore $\mathrm{S}$, Katoh $\mathrm{O}$, Matsuo $\mathrm{H}$, et al. Retrograde percutaneous recanalization of chronic total occlusion of the coronary arteries: procedural outcomes and predictors of success in contempo- 
rary practice. Circ Cardiovasc Interv 2009; 2: 124-32.

4. Kawasaki T, Koga H, Serikawa T. New bifurcation guidewire technique: a reversed guidewire technique for extremely angulated bifurcation--a case report. Catheter Cardiovasc Interv 2008; 71: 73-6.

5. Watanabe S, Saito N, Bao B, et al. Microcatheter-facilitated reverse wire technique for side branch wiring in bifurcated vessels: an in vitro evaluation. EuroIntervention 2013; 9: 870-7.

6. Werner G, Martin-Yuste V, Hildick-Smith D, et al. A randomized multicentre trial to compare revascularization with optimal medical therapy for the treatment of chronic total coronary oc- clusions. Eur Heart J 2018; 39: 2484-93.

7. Nomura T, Kikai M, Hori Y, et al. Tips of the dual-lumen microcatheter-facilitated reverse wire technique in percutaneous coronary intervention for markedly angulated bifurcated lesions. Cardiovasc Interv Ther 2018; 33: 146-53.

8. Canova P, Satogami K, Fiocca L, et al. Successful PCI of a bifurcation lesion using "Reverse Wire" technique with a duallumen microcatheter. Cath Lab Digest 2019; 27.

9. Hasegawa K, Yamamoto W, Nakabayashi S, et al. Streamlined reverse wire technique for the treatment of complex bifurcated lesions. Catheter Cardiovasc Interv 2019; 96: E287-91. 